Received: 29 December 2016

Accepted: 19 April 2017

Published online: 31 May 2017

\section{Ag-Mg antisite defect induced high thermoelectric performance of $\alpha-\mathrm{MgAgSb}$}

\author{
Zhenzhen Feng ${ }^{1}$, Jihua Zhang ${ }^{1,2}$, Yuli Yan ${ }^{1}$, Guangbiao Zhang ${ }^{1}$, Chao Wang ${ }^{1}$, Chengxiao Peng ${ }^{1}$, \\ Fengzhu Ren ${ }^{1}$, Yuanxu Wang ${ }^{1}$ \& Zhenxiang Cheng ${ }^{1,3}$
}

Engineering atomic-scale native point defects has become an attractive strategy to improve the performance of thermoelectric materials. Here, we theoretically predict that Ag-Mg antisite defects as shallow acceptors can be more stable than other intrinsic defects under Mg-poor-Ag/Sb-rich conditions. Under more Mg-rich conditions, Ag vacancy dominates the intrinsic defects. The $p$-type conduction behavior of experimentally synthesized $\alpha-M g A g S b$ mainly comes from Ag vacancies and Ag antisites (Ag on Mg sites), which act as shallow acceptors. Ag-Mg antisite defects significantly increase the thermoelectric performance of $\alpha-M g A g S b$ by increasing the number of band valleys near the Fermi level. For Li-doped $\alpha-M g A g S b$, under more Mg-rich conditions, Li will substitute on Ag sites rather than on $\mathrm{Mg}$ sites and may achieve high thermoelectric performance. A secondary valence band is revealed in $\alpha-M g A g S b$ with 14 conducting carrier pockets.

Thermoelectric materials can perform direct conversion between electrical and thermal energy. Thermoelectric performance is quantified by the figure of merit, $Z T=S^{2} \sigma T / \kappa$, where $S$ is the Seebeck coefficient, $\sigma$ is the electrical conductivity, $T$ is the absolute temperature, and $\kappa$ is the total thermal conductivity, which consists of both electronic $\left(\kappa_{e}\right)$ and lattice $\left(\kappa_{l}\right)$ components ${ }^{1-3}$. A high $Z T$ value indicates good thermoelectric properties, and one therefore should try to increase the power factor $\left(S^{2} \sigma\right)$ and decrease the thermal conductivity $\left(\kappa=\kappa_{e}+\kappa_{l}\right)$. A large power factor can be achieved by (a) increasing the density of states near the Fermi level (by forming localized resonant states ${ }^{4,5}$ or increasing band degeneracy ${ }^{6-16}$ ), and (b) by increasing the energy dependence of the carrier mobility using energy filtering ${ }^{17,18}$. Meanwhile, forming a solid solution ${ }^{19-21}$ and creating strong lattice anharmonicity ${ }^{22-28}$ can achieve low lattice thermal conductivity. A recent study has proposed that engineering atomic-scale native point defects can simultaneously optimize the thermal and electrical performances of thermoelectric materials ${ }^{29,30}$, which is becoming an attractive strategy to improve $Z T$ values. Native point defects play important roles in conduction in semiconductors, and they can change the band structure ${ }^{29}$.

The $\alpha$ phase of $\mathrm{MgAgSb}^{31}$ shows superior thermoelectric properties in the low temperature range ${ }^{31-44}$. Great efforts have been devoted to understanding and enhancing the unique thermoelectric properties of $\alpha-\mathrm{MgAgSb}$. The carrier concentration of $\alpha-\mathrm{MgAgSb}$-based materials is relatively low at room temperature, which leads to its high electrical resistivity. To overcome this limitation, extrinsic doping, including Na doping ${ }^{35}, \mathrm{Cu}^{\text {doping }}{ }^{36}$. In doping $^{38}$, and changing the $\mathrm{Sb}$ content ${ }^{41}$ have been used to increase the carrier concentration of $\alpha-\mathrm{MgAg}_{0.97} \mathrm{Sb}_{0.99}$ or $\alpha-\mathrm{MgAgSb}$, although the electrical resistivity $\left(1-4.5 \times 10^{-5} \Omega \cdot \mathrm{m}\right)$ is still larger than those of good thermoelectric materials, such as $\mathrm{CoSb}_{3}\left(0.3-1 \times 10^{-5} \Omega \cdot \mathrm{m}\right)^{45}$ and $\mathrm{Bi}_{2} \mathrm{Te}_{3}\left(1-1.5 \times 10^{-5} \Omega \cdot \mathrm{m}\right)^{46}$. Liu et al. used Li doping to increase the carrier concentration of $\mathrm{MgAg}_{0.97} \mathrm{Sb}_{0.99}$, and a high average $Z T^{39}$ of 1.1 from $300 \mathrm{~K}$ to $548 \mathrm{~K}$ was achieved.

Intrinsic defects represent another effective way to tune the carrier concentration to enhance the thermoelectric performance. Moreover, extrinsic point defects strongly influence the native point defects. Recently, Liu et al. reported that $\mathrm{Ag}$ vacancy could increase the $Z T$ for $\alpha-\mathrm{MgAgSb}^{30}$. Moreover, the Ag vacancy concentration can be tuned by the hot pressing temperature, which they denoted as the recovery effect. Therefore, it is necessary to explore the conditions for forming intrinsic defects and their influence on the electronic structure.

${ }^{1}$ Institute for Computational Materials Science, School of Physics and Electronics, Henan University, Kaifeng, 475004, China. ${ }^{2}$ Guizhou Provincial Key Laboratory of Computational Nano-Material Science, Guizhou Education University, 115 Gaoxin Road, Guiyang, 550018, China. ${ }^{3}$ Institute for Superconducting and Electronic Materials, Innovation Campus, University of Wollongong, Squires Way, North Wollongong, NSW 2500, Australia. Correspondence and requests for materials should be addressed toY.W. (email: wangyx@henu.edu.cn) or Z.C. (email: cheng@uow.edu.au) 
In this work, the chemical potentials and defect formation energies of native point defects and Li doping in $\alpha-\mathrm{MgAgSb}$ at all possible charge states are studied by using density functional theory. We found that the defect formation energies strongly depend on the chemical potentials. Ag vacancies and Ag- $\mathrm{Mg}$ antisites ( $\mathrm{Ag}$ on $\mathrm{Mg}$ sites) are the dominant defects that act as shallow acceptors, which determine the $p$-type conduction. Moreover, the $\mathrm{Ag}_{\mathrm{Mg}}$ point defect in $\alpha-\mathrm{MgAgSb}$ may have higher $Z T$ than the Ag vacancy. For Li-doped $\alpha-\mathrm{MgAgSb}$, the doping formation energies strongly depend on the chemical potentials. Under more Mg-rich conditions, Li will substitute on $\mathrm{Ag}$ sites $\left(\mathrm{Li}_{\mathrm{Ag}}\right)$ rather than on $\mathrm{Mg}$ sites $\left(\mathrm{Li}_{\mathrm{Mg}}\right)$, and a larger $Z T$ can be achieved by $\mathrm{Li}_{\mathrm{Ag}}$ doping than by $\mathrm{Li}_{\mathrm{Mg}}$ doping. By reasonably controlling the chemical potential, both the antisite defect $\mathrm{Ag}_{\mathrm{Mg}}$ and Li substitution on $\mathrm{Ag}$ sites of $\alpha-\mathrm{MgAgSb}$ can be obtained, and the products may be promising thermoelectric materials for low temperature power generation.

\section{Results and Discussion}

Chemical potentials and formation energies of native point defects. Engineering intrinsic defects may be an effective way to improve the thermoelectric performance of $\alpha-\mathrm{MgAgSb}$. Due to the complex phase transitions and the appearance of secondary phases, previous experimental works have shown that it is difficult to synthesize pure phase $\alpha-\mathrm{MgAgSb}$. Different types of native point defects may easily appear in $\alpha-\mathrm{MgAgSb}$. Thus, it is necessary to first explore the conditions for forming intrinsic point defects in $\alpha$-MgAgSb.

The defect formation energy $\left(\Delta H_{f}\right)$ is defined as

$$
\Delta H_{f}=E_{\text {defect }}^{q}-E_{\text {perfect }}+\sum_{i} n_{i} \mu_{i}+q\left(E_{F}+E_{V}+\Delta V\right),
$$

where $E_{\text {defect }}^{q}$ is the total energy of the supercell with the incorporated defect, $E_{\text {perfect }}$ is the total energy of the supercell without the incorporated defect, $n_{i}$ is the number of atoms being removed or added, and $\mu_{i}$ is the corresponding chemical potential, $E_{F}$ is the Fermi energy, $E_{V}$ is the energy with respect to the valence band maximum (VBM), and $\Delta V$ is the average difference between the local potentials far from the defect in the defective supercell and the corresponding ones in the perfect supercell ${ }^{47}$.

We calculated the accessible range of chemical potentials for the equilibrium growth conditions of $\alpha-\mathrm{MgAgSb}$. Under equilibrium conditions for the crystal growth, the steady production of the host material, $\alpha-\mathrm{MgAgSb}$, should satisfy the following equations:

$$
\begin{gathered}
\mu_{M g A g S b}=\mu_{M g}+\mu_{A g}+\mu_{S b}, \\
E_{M g A g S b}=E_{M g}+E_{A g}+E_{S b}+\Delta H_{f}(M g A g S b), \\
\Delta \mu_{M g}+\Delta \mu_{A g}+\Delta \mu_{S b}=\Delta H_{f}(M g A g S b),
\end{gathered}
$$

where $\mu_{M g}, \mu_{A g}$, and $\mu_{S b}$ are the chemical potentials of $\mathrm{Mg}$, $\mathrm{Ag}$, and Sb, respectively, and $\Delta H_{f}(M g A g S b)$ is the formation energy for $\alpha$-MgAgSb. To avoid the precipitation of source elements, $\Delta \mu_{M g}, \Delta \mu_{A g}$, and $\Delta \mu_{S b}$ should satisfy:

$$
\Delta \mu_{M g}<0, \Delta \mu_{A g}<0, \Delta \mu_{S b}<0 .
$$

To maintain the stability of $\mathrm{MgAgSb}$ during growth and avoid any competing phases (such as $\mathrm{MgAg}, \mathrm{Mg}_{3} \mathrm{Sb}_{2}$, and $\mathrm{Ag}_{3} \mathrm{Sb}$ ), the chemical potential s $\Delta \mu_{M g}, \Delta \mu_{A g}$, and $\Delta \mu_{S b}$ must satisfy the following limits:

$$
\begin{gathered}
\Delta \mu_{M g}+\Delta \mu_{A g}<\Delta H_{f}(M g A g), \\
3 \Delta \mu_{M g}+2 \Delta \mu_{S b}<\Delta H_{f}\left(M g_{3} S b_{2}\right), \\
3 \Delta \mu_{A g}+\Delta \mu_{S b}<\Delta H_{f}\left(A g_{3} S b\right) .
\end{gathered}
$$

All calculated heats of formation of ternary and binary compounds in this work are given per formula unit.

Equations (4)-(8) can be projected onto the two-dimensional plane with two independent variables, $\Delta \mu_{M g}$ and $\Delta \mu_{A g}$, as shown in Fig. 1. The shaded region represents the area for the equilibrium growth conditions of $\alpha-\mathrm{MgAgSb}$. Figure 1 asserts that $\alpha-\mathrm{MgAgSb}$ is only thermodynamically stable within a narrow $\mathrm{Mg}$-Ag compositional range. The thermodynamically stable ranges of chemical potentials for the elements in $\alpha-\mathrm{MgAgSb}$ are obtained by excluding the regions of chemical potentials in which competing phases are thermodynamically stable. Here, we present the calculated values at two representative chemical potential points labeled as $\mathrm{A}$ $(-0.69 \mathrm{eV}, 0,0)$ and $\mathrm{B}(-0.469 \mathrm{eV},-0.032 \mathrm{eV},-0.162)$ in Fig. 1 for $\Delta \mu_{M g}, \Delta \mu_{A g}$, and $\Delta \mu_{S b}$, respectively.

To predict the conductivity type of $\mathrm{MgAgSb}$ with intrinsic defects, we calculated the Fermi level pinning positions. Figure 2(a) and (b) shows the calculated formation energies of native point defects as a function of the Fermi levels at chemical potential points $\mathrm{A}$ and $\mathrm{B}$, respectively. The calculated transition energies for these defects are shown in Fig. 3. From the single-particle energy point of view, $\mathrm{V}_{\mathrm{Mg}}, \mathrm{V}_{\mathrm{Ag}}, \mathrm{Sb}_{\mathrm{I}}$, and $\mathrm{Ag}_{\mathrm{Mg}}$ should be acceptor-like defects, whereas $\mathrm{V}_{\mathrm{Sb}}, \mathrm{Mg}_{\mathrm{I}}, \mathrm{Ag}_{\mathrm{I}}$, and $\mathrm{Mg}_{\mathrm{Ag}}$ should be donor-like defects. Under $\mathrm{Mg}$-poor- $\mathrm{Ag} / \mathrm{Sb}$-rich conditions (point $\mathrm{A}$ in Fig. 1), the formation energy of the $\mathrm{Ag}_{\mathrm{Mg}}$ antisite defect is very low, meaning that it is the dominant 


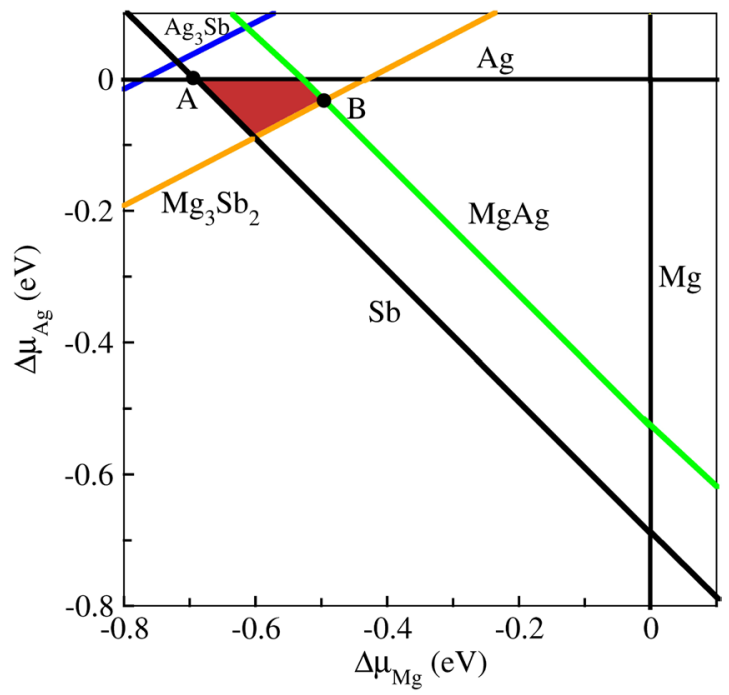

Figure 1. Accessible range of chemical potentials (shaded region) for equilibrium growth conditions of $\alpha-M g A g S b$. The specific points A and B were chosen for the representative chemical potentials to be used for the following doping formation energy calculations.
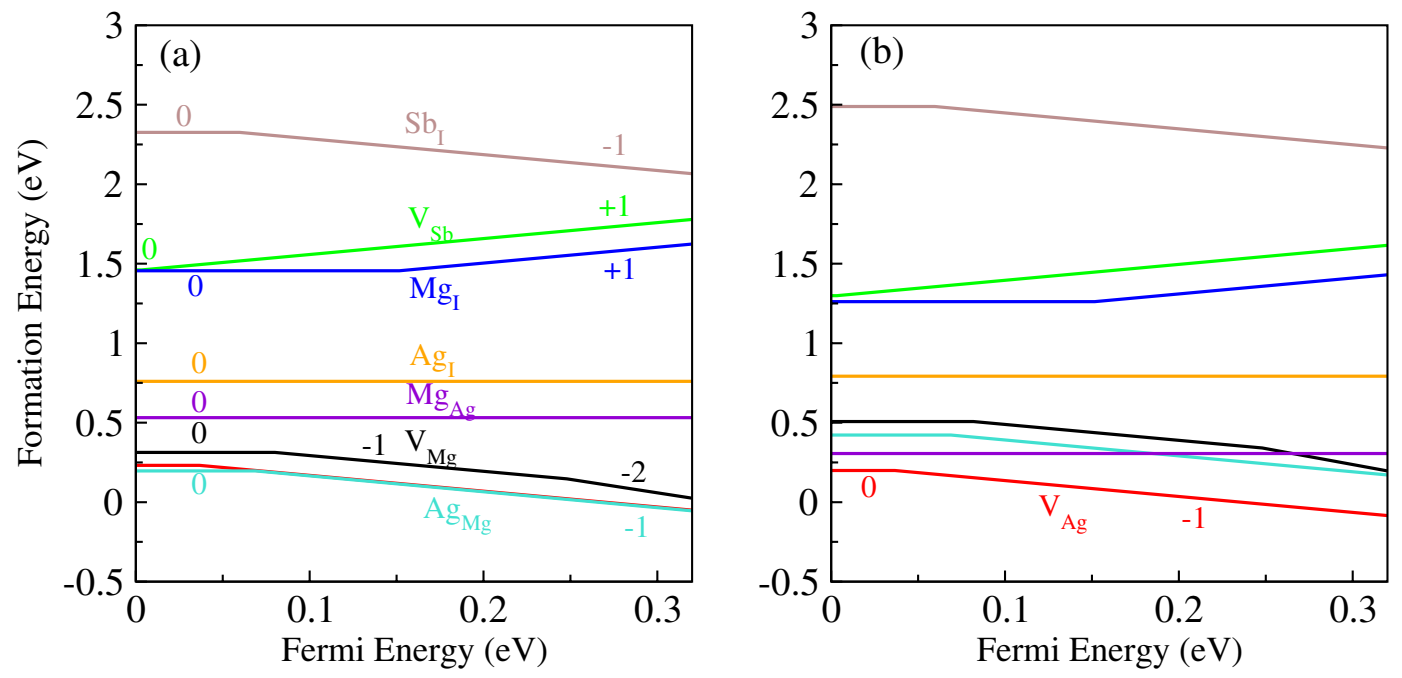

Figure 2. The calculated formation energies of native point defects in $\alpha-\mathrm{MgAgSb}$ as a function of the Fermi level, with chemical potentials at (a) point $\mathrm{A}$ and (b) point B.

acceptor, and p-type conductivity can be realized by forming $\mathrm{Ag}_{\mathrm{Mg}}$ antisite defects. The $\mathrm{Ag}_{\mathrm{Mg}}$ antisite defect is thermodynamically stable. This suggests that $\mathrm{Ag}_{\mathrm{Mg}}$ may stably exist in an $\mathrm{Mg}$ poor environment. Under more Mg-rich conditions (point B in Fig. 1), the $\mathrm{V}_{\mathrm{Ag}}$ defect has the lowest formation energy, indicating that it is now the dominant type of acceptor, which is consistent with the results reported by Liu et al. ${ }^{30}$. Thus, our calculation results for the formation energy can explain why $\alpha-\mathrm{MgAgSb}$ often exhibits $p$-type conductivity. From Fig. 3, it is seen that the transition energies of $\mathrm{V}_{\mathrm{Ag}}$ and $\mathrm{Ag}_{\mathrm{Mg}}$ are $0.036 \mathrm{eV}$ and $0.068 \mathrm{eV}$ above the $\mathrm{VBM}$, respectively, indicating that $\mathrm{V}_{\mathrm{Ag}}$ and $\mathrm{Ag}_{\mathrm{Mg}}$ are shallow acceptors. On the other hand, all the defects that create deep levels, such as $\mathrm{Mg}_{\mathrm{I}}$ and $\mathrm{V}_{\mathrm{Mg}}$, have higher formation energies. Thus, the formation energies of the native point defects strongly depend on the chemical potentials, and $\mathrm{Ag}_{\mathrm{Mg}}$ antisites and $\mathrm{Ag}$ vacancies are the dominant acceptor defects in $\alpha-\mathrm{MgAgSb}$. The calculated formation energy using chemical potentials is close to the real preparation environment. Under the different circumstances, we can compare the types of doping with which conditions are easier or more difficult to achieve, which can explain the experimental phenomena and provide a reference for controlling the defect type.

Effects of native defects on electronic structure. Miao et al. have calculated the band structure of $\alpha-\mathrm{MgAgSb}$ using the Perdew-Burke-Ernzerhof generalized gradient approximation (GGA) (GGA-PBE) exchange-correlation functional in the Vienna ab-initio simulation package (VASP), and predicted that $\alpha-\mathrm{MgAgSb}_{\text {is a semimetal }}{ }^{37}$. Using the local density approximation (LDA) exchange-correlation potential as 


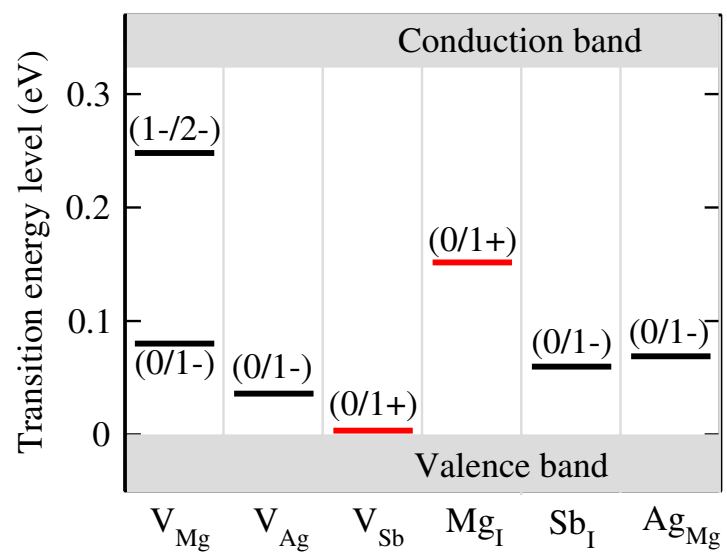

Figure 3. The calculated transition energy levels of various intrinsic defects in $\alpha-\mathrm{MgAgSb}$. The black bars show the acceptor levels, and the red bars show the donor levels.

(a)

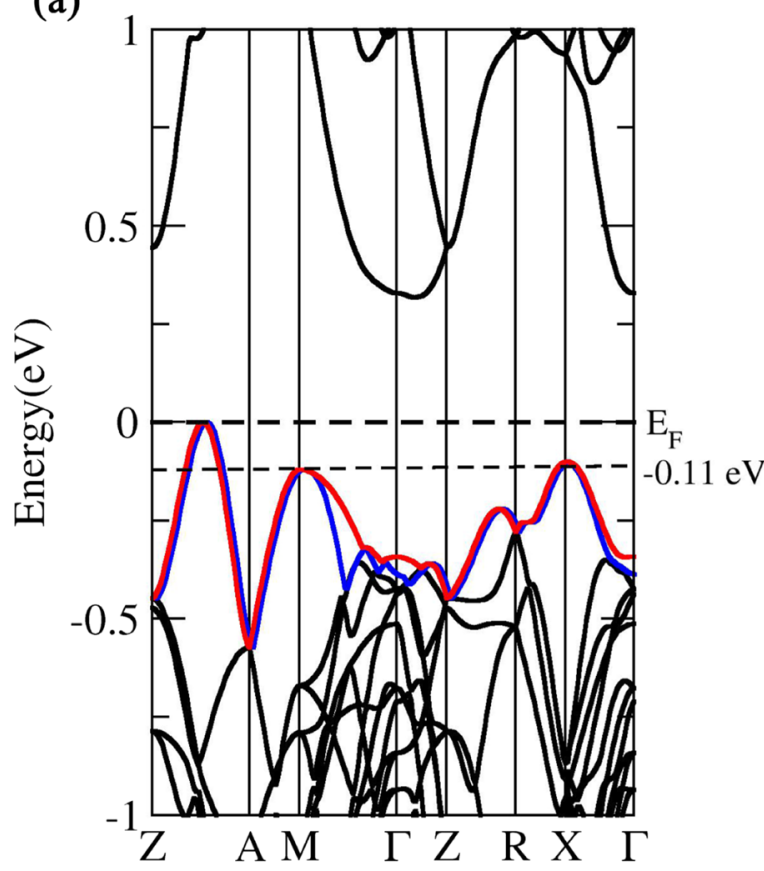

(b)

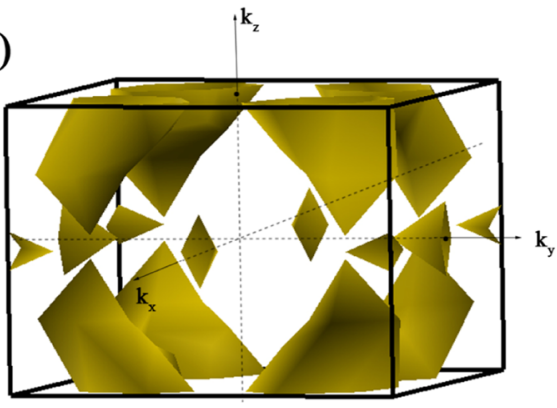

(c)

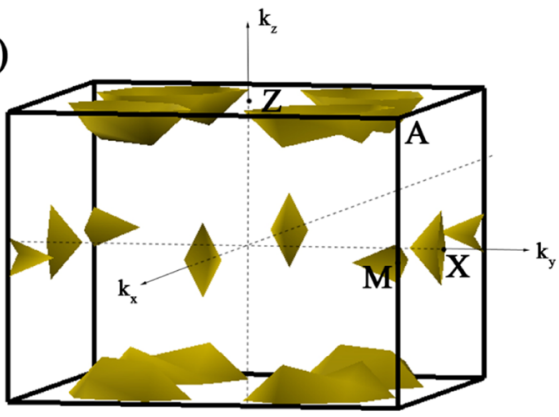

Figure 4. (a) Calculated band structure of $\alpha-\mathrm{MgAgSb}$. The top of the valence band is set to zero. The dashed lines denote the Fermi level. The special $k$ points $Z, A, M, \Gamma, R$, and $X$ are located at the points $(0,0,0.5),(0.5$, $0.5,0.5),(0.5,0.5,0),(0,0,0),(0,0.5,0.5)$, and $(0,0.5,0)$, respectively. (b) Fermi surface calculated for a Fermi level at $-0.11 \mathrm{eV}$ below the valence band maximum, showing the 7 pockets of the red valence band in (a). (c) Fermi surface calculated for a Fermi level at $-0.11 \mathrm{eV}$ below the valence band maximum, showing the 7 pockets of the blue valence band in (a).

implemented in the VASP, Ying et al. found that $\alpha-\mathrm{MgAgSb}$ is a semiconductor with an indirect band gap of $0.1 \mathrm{eV}^{38}$. Because the GGA and LDA exchange correlation potentials always underestimate the band gap of crystals, we calculated the band structure of $\alpha-\mathrm{MgAgSb}$ as implemented in WIEN2k with the Tran-Blaha modified Becke-Johnson (TB-mBJ) exchange correlation potential, as is shown in Fig. 4(a). Because the electron transport is closely related to the electronic states near the valence band maximum (VBM) and conduction band minimum (CBM), we only focused on the electronic states near the Fermi level. As shown in Fig. 4(a), $\alpha$-MgAgSb is a semiconductor with a band gap of $0.32 \mathrm{eV}$, and the band structure is characterized by an indirect band gap, with the $\mathrm{CBM}$ near the $\Gamma$ point and the VBM between $\mathrm{Z}$ and $\mathrm{A}$. Sheng et al. also calculated the band structure by using the $\mathrm{mBJ}$ functional and the Heyd-Scuseria-Ernzerhof (HSE) approach as implemented in VASP ${ }^{42}$. They did not consider the $k$-path of Z-A, however, so they thought that the VBM was at X. As shown in Fig. 4(a), the VBM should be located at Z-A, and the maximum of the second valence band is located at $\mathrm{M}$ and $\mathrm{X}$. The top of the 
(a)

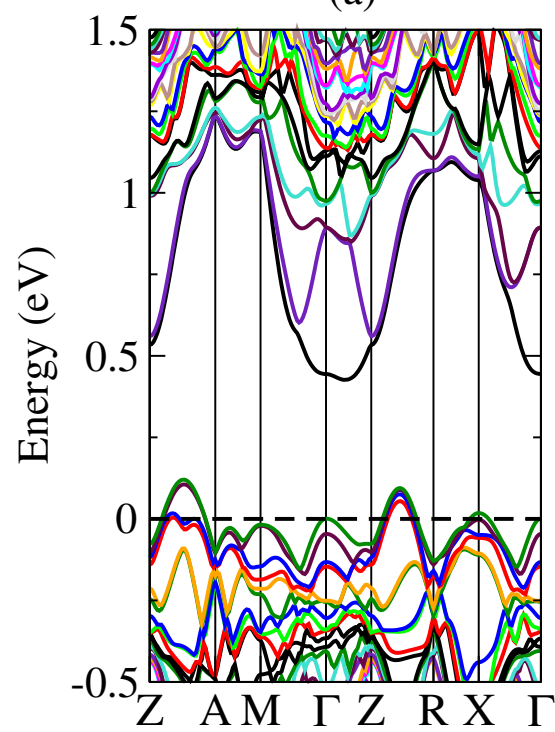

(b)

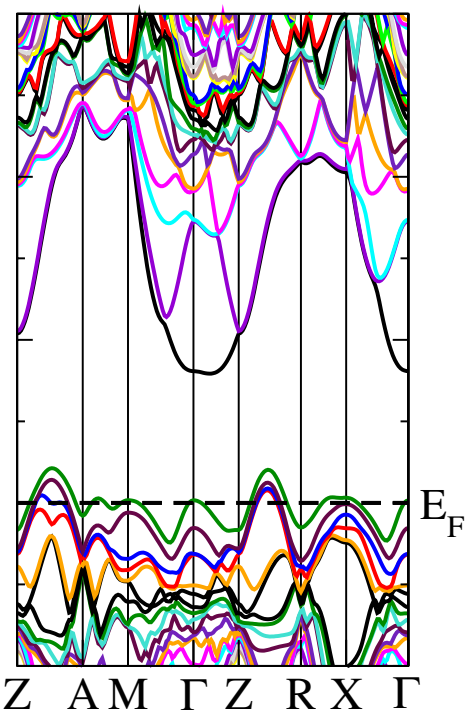

Figure 5. Calculated band structures of (a) $\mathrm{Mg}_{48} \mathrm{Ag}_{47} \mathrm{Sb}_{48}$ with $\mathrm{Ag}$ vacancy, and (b) $\mathrm{Mg}_{47} \mathrm{Ag}_{49} \mathrm{Sb}_{48}$ with $\mathrm{Ag}$ antisite. The top of the valence band is set to zero.

valence band has a stronger dispersion than the bottom of the conduction band. The band dispersion relationship determines the effective mass, and the band mass of a single valley can be obtained by the following:

$$
m^{*}=\hbar^{2}\left[\frac{\mathrm{d}^{2} E(k)}{\mathrm{d} k}\right]_{E(k)=E_{F}}^{-1}
$$

where $k$ is the wave vector, $E_{\mathrm{F}}$ is the Fermi energy, and $\hbar$ is the reduced Planck's constant. According to Eq. (9), we know that the band effective mass at the top of the valence band is smaller than that at the bottom of the conduction band. Such large band dispersion of the valence band is conducive to the transmission of electrons. The small effective mass of top valence bands is helpful for increasing the electrical conductivity of $p$-type $\alpha-\mathrm{MgAgSb}$, although electrical conductivity is also determined by the carrier concentration.

As is well known, the maximum $Z T$ of a material depends on the dimensionless thermoelectric quality factor $B \propto \mu m_{D O S}^{* 3 / 2} / \kappa_{L}^{48}$, where $\mu$ is the mobility of the carrier and $m_{D O S}^{*}$ is the density-of-states (DOS) effective mass. The relationship between the density of states effective mass, the band degeneracy, $N_{V}$, and the band effective mass, $m_{b}^{*}$, is given by: $m_{D O S}^{*}=N_{V}^{2 / 3} m_{b}^{*}$. If acoustic phonon scattering dominates the carrier transport, then $\mu \propto 1 / m_{b}^{* 3 / 2} m_{I}^{*}$ and $B \propto N_{V} / m_{I}^{*} \kappa_{L}$, where $m_{I}^{*}$ is the inertial mass. Thus, a large $N_{V}$ is beneficial to a large $m_{D O S}^{*}$ without deterioration of $\mu^{6}$. The band degeneracy $N_{V}$ is based on the effective total number of independent carrier pockets or valleys in the Brillouin zone, including both orbital and symmetry related degeneracy. We adopted the strategy of increasing $N_{V}$ for a high $Z T$ as an example, as was well demonstrated for $\mathrm{PbTe}^{6}$. As a result of heavy hole doping and relatively light bands at the VBM, the Fermi level quickly moves down into the valence band, allowing a large population of holes to form in the secondary valence band. The calculations show that the secondary VBM is located at about $-0.11 \mathrm{eV}$ below the VBM. The Fermi surface calculations for a Fermi level $-0.11 \mathrm{eV}$ below the VBM of the red valence band and of the blue valence band are shown in Fig. 4(b) and (c), respectively. Figure 4(b) shows 8 half-pockets along Z-A, 4 quarter pockets at the M point, and 4 half-pockets at the $\mathrm{X}$ point so that the full number of valleys is 7 . Figure 4(c) also shows that the full number of valleys is 7. Therefore, the iso-energy Fermi surface for an energy level at $-0.11 \mathrm{eV}$ has a high degeneracy with 14 isolated pockets. The large band degeneracy $N_{\mathrm{V}}$ may contribute to the high Seebeck coefficient at relatively high carrier concentrations. Based on above analysis, the large band dispersion of the valence band, together with the high band degeneracy with $N_{V}=14$, may be the most significant feature that contributes to the good thermoelectric performance of $p$-type heavily doped $\alpha$-MgAgSb.

Figure 5(a) and (b) shows the band structures of $\mathrm{Mg}_{48} \mathrm{Ag}_{47} \mathrm{Sb}_{48}$ with an $\mathrm{Ag}$ vacancy and $\mathrm{Mg}_{47} \mathrm{Ag}_{49} \mathrm{Sb}_{48}$ with an $\mathrm{Ag}_{\mathrm{Mg}}$ antisite, respectively. Both the $\mathrm{Ag}$ vacancy and the $\mathrm{Ag}$ antisite can break the symmetry of the supercell when introduced into the system. $\mathrm{V}_{\mathrm{Ag}}$ and $\mathrm{Ag}_{\mathrm{Mg}}$ show typical $p$-type doping behavior by shifting the Fermi level into the valence bands. The number of band valleys near the Fermi level increases. The large band degeneracy $N_{\mathrm{V}}$ and the heavy band effective mass can jointly contribute to the high Seebeck coefficient. Moreover, for $p$-type $\alpha-\mathrm{MgAgSb}$, the carrier concentration largely depends on the number of band valleys near the Fermi level. A high carrier concentration may help to increase the electrical conductivity. Therefore, the native defects $\mathrm{V}_{\mathrm{Ag}}$ and $\mathrm{Ag} \mathrm{Mg}_{\mathrm{gay}}$ play an important role in achieving a higher Seebeck coefficient and higher electrical conductivity, which will lead to a large $Z T$ for $\alpha-M g A g S b . \alpha-M g A g S b$ with the $\mathrm{Ag}_{\mathrm{Mg}}$ defect has a larger number of band valleys near the Fermi level than with the $\mathrm{V}_{\mathrm{Ag}}$ defect, which may lead to a larger $Z T$ than with $\mathrm{V}_{\mathrm{Ag}}$. 

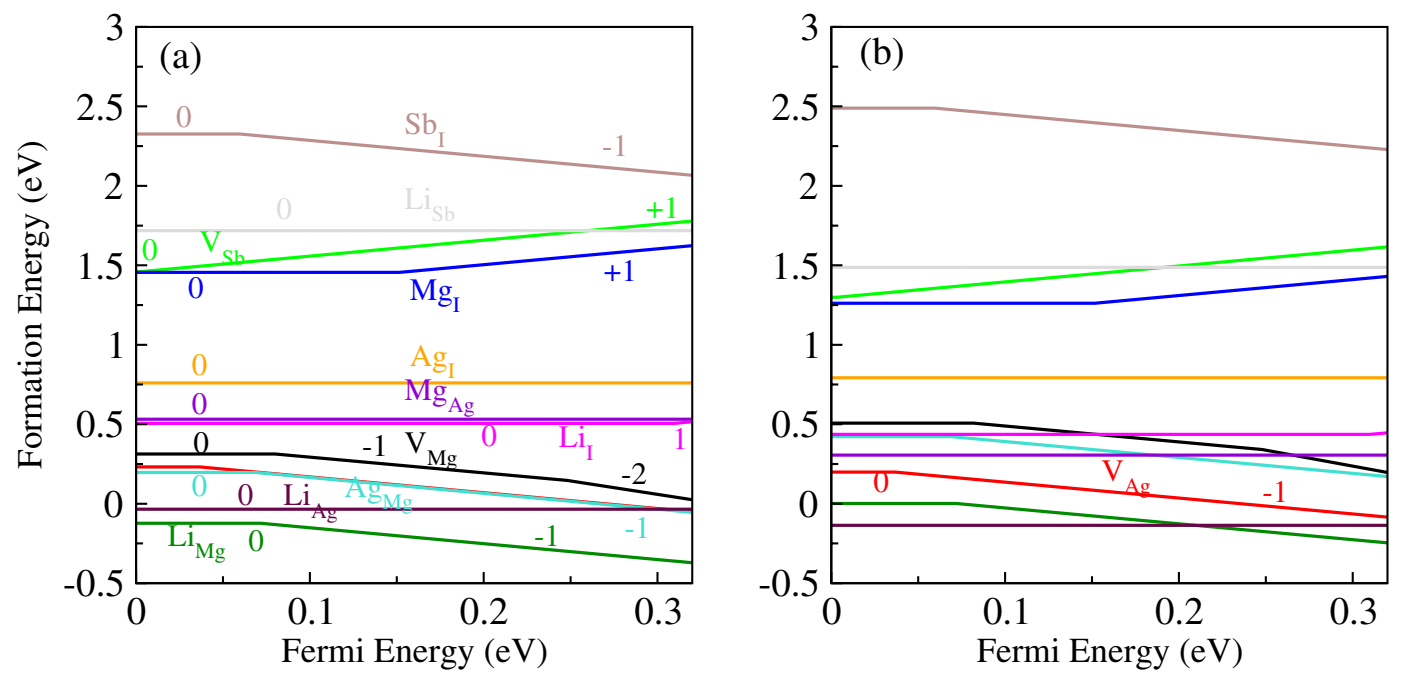

Figure 6. The calculated formation energies for Li doping and various native point defects in $\alpha-\mathrm{MgAgSb}$ as a function of the Fermi level, with chemical potentials at (a) point A and (b) point $B$.

Li-doped $\alpha-M g A g S b . \quad P r e v i o u s ~ e x p e r i m e n t a l$ work showed that Li doping can significantly increase the $Z T$ value of $\alpha-\mathrm{MgAg}_{0.97} \mathrm{Sb}_{0.99}{ }^{39}$. After Li doping, the carrier concentration increased from $2.3 \times 10^{19} \mathrm{~cm}^{-3}$ to $1.4 \times 10^{20} \mathrm{~cm}^{-3}$. The achieved average $Z T$ was 1.1 from 300 to $548 \mathrm{~K}$. The authors noted that Li was substituted onto the $\mathrm{Mg}$ sites ${ }^{39}$. It is valuable to explore how the chemical potential affects the doping sites in Li-doped $\alpha-\mathrm{MgAgSb}$. We calculated the formation energies of Li-doped $\alpha-\mathrm{MgAgSb}$ as a function of chemical potential. For Li doping, the chemical potentials of impurities should satisfy other constraints to avoid the formation of impurity-related phases (such as $\mathrm{Li}$ source element, $\mathrm{LiAg}, \mathrm{Li}_{2} \mathrm{Sb}$, or $\mathrm{Li}_{3} \mathrm{Sb}$ ):

$$
\begin{gathered}
\Delta \mu_{L i}<0, \\
\Delta \mu_{L i}+\Delta \mu_{A g}<\Delta H_{f}(\operatorname{LiAg}), \\
2 \Delta \mu_{L i}+\Delta \mu_{S b}<\Delta H_{f}\left(L i_{2} S b\right), \\
3 \Delta \mu_{L i}+\Delta \mu_{S b}<\Delta H_{f}\left(L i_{3} S b\right) .
\end{gathered}
$$

Based on the representative chemical potential points, we can calculate the chemical potential of $\mathrm{Li}$, and the values of the chemical potentials at points $\mathrm{A}$ and $\mathrm{B}$ are $-0.8857 \mathrm{eV}$ and $-0.8163 \mathrm{eV}$ for $\Delta \mu_{L i}$, respectively. Then, the chemical potential is used for calculating the formation energy for Li-related defects. The impurities can either be at interstitial sites or substitute for $\mathrm{Mg}$, Ag, or Sb. Therefore four different point defects, $\mathrm{Li}_{\mathrm{Mg}}, \mathrm{Li}_{\mathrm{Ag}}, \mathrm{Li}_{\mathrm{Sb}}$, and $\mathrm{Li}_{\mathrm{I}}$, have been included in our calculation. Because of the large formation energy for $\mathrm{Li}_{\mathrm{Sb}}$, we only show the $\mathrm{Li}_{\mathrm{Sb}}$ with zero charges.

The calculated impurity formation energies of the doping systems are plotted in Fig. 6. As shown in Fig. 6, formation energies strongly depend on the chemical potentials. The thermodynamic transition level between $\mathrm{Li}_{\mathrm{I}}^{0}$ and $\mathrm{Li}_{\mathrm{I}}^{-1}$ is $0.01 \mathrm{eV}$ below the CBM, indicating that $\mathrm{Li}_{\mathrm{I}}$ is a shallow donor. At point $\mathrm{A}$, substitutional $\mathrm{Li}$ on $\mathrm{Mg}$ sites has the lowest formation energy. At point B, substitutional Li on Ag sites has much lower formation energy than on $\mathrm{Mg}$ sites. Thus, at point $\mathrm{A}, \mathrm{Li}$ doping can lead to good p-type conductivity, while at point $\mathrm{B}$, Li doping cannot change the conductivity type of $\alpha-\mathrm{MgAgSb}$ because of $\mathrm{Li}_{\mathrm{Ag}}$ with zero charges. The formation energy for Li substitution on $\mathrm{Mg}$ sites at point $\mathrm{A}$ is smaller than for substituting $\mathrm{Li}$ atoms on $\mathrm{Ag}$ sites or $\mathrm{Sb}$ sites, implying that the $\mathrm{Li}$ atoms prefer to occupy the $\mathrm{Mg}$ sites rather than the $\mathrm{Ag}$ or $\mathrm{Sb}$ sites at point $\mathrm{A}$. On the other hand, at point $\mathrm{B}$ (more Mg-rich conditions), Li substitution on Ag sites of $\alpha-\mathrm{MgAgSb}$ is the most stable structure. Therefore, we can substitute $\mathrm{Li}$ on $\mathrm{Mg}$ sites or $\mathrm{Ag}$ sites in $\alpha-\mathrm{MgAgSb}$ by controlling the chemical potential of Li under different conditions.

We also investigated the electronic structure and the transport properties for $\alpha-\mathrm{MgAgSb}$ with Li doping on $\mathrm{Mg}$ sites and $\mathrm{Ag}$ sites at a doping level of 0.02 . The calculated band structures of $\mathrm{Mg}_{47} \mathrm{LiAg}_{48} \mathrm{Sb}_{48}$ and $\mathrm{Mg}_{48} \mathrm{Ag}_{47} \mathrm{LiSb}_{48}$ are shown in Fig. 7(a) and (b), respectively. As shown in Fig. 7(a), the most obvious change from substituting $\mathrm{Li}$ on $\mathrm{Mg}$ sites is the appearance of large valley degeneracy in the valence bands near the Fermi level, and the band gap of $\mathrm{Li}$-doped $\alpha-\mathrm{MgAgSb}$ is $0.32 \mathrm{eV}$. The good thermoelectric properties of a thermoelectric material depend on the weighted carrier mobility, $\mu\left(m_{D O S}^{*} / m_{e}\right)^{3 / 2}$; the density of states effective mass is defined by $m_{D O S}^{*}=N_{V}^{2 / 3} m_{b}^{*}$. Note that the carrier mobility is strongly affected by the band mass of a single valley: $\mu \propto 1 / m_{b}^{* 5 / 249}$. Therefore, increasing the band mass should be detrimental to the carrier mobility. Multiple degenerate valleys may produce a large $m_{D O S}^{*}$ without explicitly reducing the carrier mobility. In that case, a large 
(a)

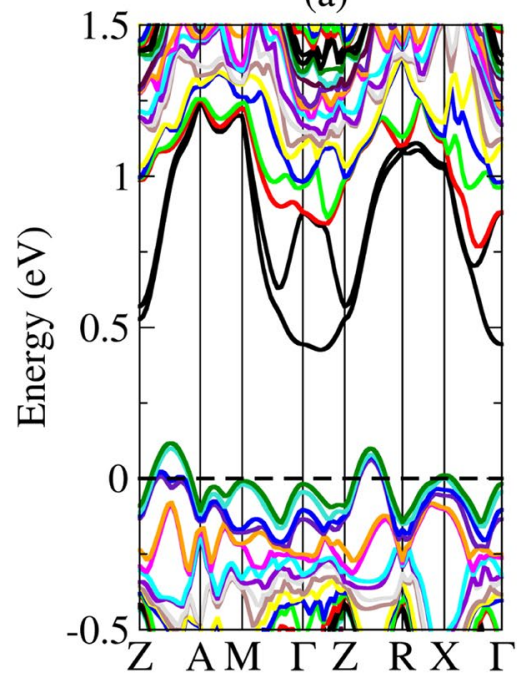

(b)

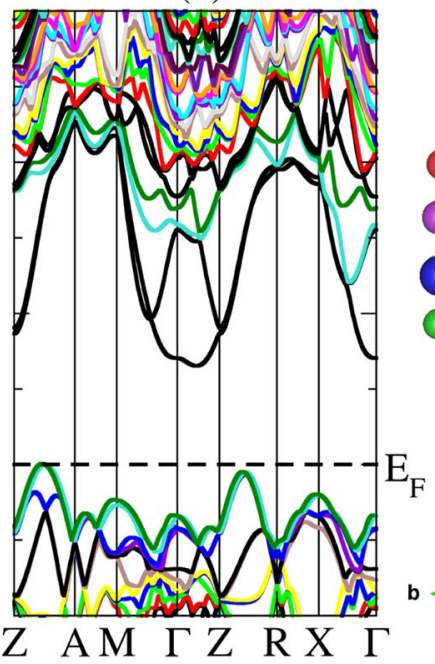

(C)

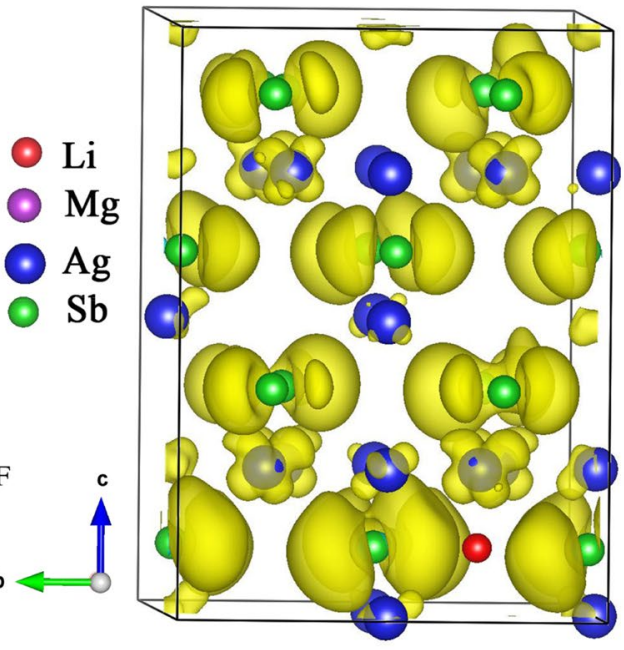

Figure 7. Calculated band structures of (a) $\mathrm{Mg}_{47} \mathrm{LiAg}_{48} \mathrm{Sb}_{48}$ and (b) $\mathrm{Mg}_{48} \mathrm{Ag}_{47} \mathrm{LiSb}_{48}$. The dashed line denotes the Fermi level. (c) Calculated band-decomposed charge density of $\mathrm{Mg}_{0.98} \mathrm{Li}_{0.02} \mathrm{AgSb}$ for valence bands near the Fermi level at point $\Gamma$, with the isosurface value of $0.0009 \mathrm{e}^{-3}$.

valley degeneracy is helpful for the thermoelectric material ${ }^{6}$. As can be seen in Fig. 7(a), the Fermi level moves down into the valence band by $0.11 \mathrm{eV}$ because of Li doping on $\mathrm{Mg}$ sites in $\alpha-\mathrm{MgAgSb}$, and the energy of the $\Gamma$ point becomes higher towards the Fermi level so that the number of band valleys near the Fermi level increases.

To explain the reason why the $\Gamma$ point becomes higher and moves toward the Fermi level, we calculated the partial charge densities near the Fermi level at the $\Gamma$ point using VASP, as shown in Fig. 7(c). Because there is little charge density distribution around the $\mathrm{Mg}$ atoms, we do not display the $\mathrm{Mg}$ atoms. From the shape of the charge density, we can see that the states near the Fermi level at the $\Gamma$ point mainly come from the Sb $p$ orbitals. The large band degeneracy $N_{\mathrm{V}}$ and heavy band effective mass can jointly contribute to the high Seebeck coefficient. Moreover, for $p$-type $\alpha$-MgAgSb, the carrier concentration largely depends on the number of band valleys near the Fermi level, which is mainly due to the fact that more carriers can be activated across the band gap. High carrier concentration may help to increase the electrical conductivity. Therefore, Li doping may play an important role in achieving a higher Seebeck coefficient and electrical conductivity, which will lead to a large ZT for $\mathrm{Mg}_{47} \mathrm{LiAg}_{48} \mathrm{Sb}_{48}$. Recently, Liu et al. used Li doping to increase the carrier concentration of $\mathrm{MgAg}_{0.97} \mathrm{Sb}_{0.99}$, thus decreasing the electrical resistivity, and a high average $Z T^{39}$ of 1.1 from $300 \mathrm{~K}$ to $548 \mathrm{~K}$ was achieved.

The calculated band structure of $\mathrm{Mg}_{48} \mathrm{Ag}_{47} \mathrm{LiSb}_{48}$ is shown in Fig. 7(b). As can be seen in Fig. 7(b), the number of band valleys near the Fermi level increases because the energy along Z-R becomes higher towards the Fermi level. Multiple degenerate valleys may produce a large $m_{D O S}^{*}$, and a large $m_{D O S}^{*}$ may lead to a large Seebeck coefficient.

Elastic and thermal properties. Ying et al. found that the appearance of three-centered $\mathrm{Mg}-\mathrm{Ag}-\mathrm{Sb}$ bonds in $\alpha-\mathrm{MgAgSb}$ results in low intrinsic lattice thermal conductivity ${ }^{50}$. To investigate the elastic properties of $\alpha-\mathrm{MgAgSb}$ with intrinsic defects and Li doping, the stress-strain method was used to calculate the elastic constants and other elastic properties ${ }^{51}$. A small finite strain is applied on the optimized structure, and then the atomic positions are optimized. The elastic constants are obtained from the stress of the strained structure. The calculated elastic constants of $\mathrm{MgAgSb}$ and $\mathrm{Mg}_{0.98} \mathrm{Li}_{0.02} \mathrm{AgSb}$ are listed in Table 1 . It is clearly seen that all the studied compounds satisfy the mechanical stability criteria ${ }^{52}$, indicating that they are elastically stable. On the other hand, the positive eigenvalues of the elastic constant matrix for each compound further prove that they are elastically stable. From the calculated elastic constants $C_{\mathrm{ij}}$, the polycrystalline bulk modulus $B$ and shear modulus $G$ were estimated using the Voigt-Reuss-Hill approximation ${ }^{53}$. A high (low) $B / G$ ratio of a material indicates that it is ductile (brittle), and the critical value is about $1.75^{54}$. The calculated $B / G$ ratios for $\mathrm{MgAgSb}$ and $\mathrm{Mg}_{0.98} \mathrm{Li}_{0.02} \mathrm{AgSb}$ are larger than the critical value (1.75), indicating that they are all ductile materials.

Thermal conductivity of a material includes both electronic and lattice thermal conductivity. The electronic contribution to the thermal conductivity is described by the Wiedemann-Franz relation, $\kappa_{e}=L T \sigma$, where $\mathrm{L}$ is the Lorenz number. Above the Debye temperature, the lattice thermal conductivity is generally limited by Umklapp scattering, which leads to $\kappa_{l} \propto 1 / T$. This $1 / T$ decay can only continue, however, until the minimum lattice thermal conductivity $\left(\kappa_{\min }\right)$ is reached, as defined by Cahill ${ }^{55,56}$. At high temperature $\left(T>\Theta_{D}\right), \kappa_{\min }$ can be approximated by the following formula: 


\begin{tabular}{|l|l|l|l|l|l|l|l|l|l|l|l|l|l|}
\hline & $\mathbf{C}_{11}$ & $\mathbf{C}_{12}$ & $\mathbf{C}_{13}$ & $\mathbf{C}_{33}$ & $\mathbf{C}_{44}$ & $\mathbf{C}_{66}$ & $\boldsymbol{\rho}$ & $\mathbf{B}$ & $\mathbf{G}$ & $\boldsymbol{\nu}_{\mathbf{s}}$ & $\boldsymbol{\nu}_{1}$ & $\Theta_{\mathbf{D}}$ & $\boldsymbol{\kappa}_{\min }$ \\
\hline $\mathrm{MgAgSb}$ & 89 & 34 & 47 & 70 & 30 & 18 & 6.31 & 56.2 & 26.2 & 2.04 & 3.80 & 241 & 0.55 \\
\hline $\mathrm{V}_{\mathrm{Ag}}$ & 87 & 32 & 49 & 70 & 27 & 14 & 6.08 & 56.5 & 23.6 & 1.97 & 3.80 & 231 & 0.53 \\
\hline $\mathrm{Ag}_{\mathrm{Mg}}$ & 90 & 36 & 48 & 72 & 28 & 17 & 6.18 & 58.2 & 25.6 & 2.03 & 3.86 & 239 & 0.54 \\
\hline $\mathrm{Li}_{\mathrm{Mg}}$ & 87 & 36 & 48 & 68 & 27 & 16 & 6.13 & 56.4 & 23.9 & 1.97 & 3.79 & 232 & 0.53 \\
\hline $\mathrm{Li}_{\mathrm{Ag}}$ & 93 & 35 & 49 & 69 & 29 & 16 & 6.09 & 57.9 & 25.3 & 2.04 & 3.88 & 239 & 0.54 \\
\hline
\end{tabular}

Table 1. Calculated elastic constants $\left(C_{\mathrm{ij}}\right.$ in $\left.\mathrm{GPa}\right)$, theoretical density $\left(\rho \mathrm{ing} / \mathrm{cm}^{3}\right)$, bulk modulus ( $B$ in $\mathrm{GPa})$, shear modulus ( $G$ in GPa), shear sound velocity $\left(v_{s}\right.$ in $\left.\mathrm{km} / \mathrm{s}\right)$, longitudinal sound velocity $\left(v_{l}\right.$ in $\left.\mathrm{km} / \mathrm{s}\right)$, Debye temperature $\left(\Theta_{\mathrm{D}}\right.$ in $\left.\mathrm{K}\right)$, and minimum lattice thermal conductivity $\left(\kappa_{\min }\right.$ in $\left.\mathrm{W} / \mathrm{mK}\right)$ of $\alpha-\mathrm{MgAgSb}, \mathrm{V}_{\mathrm{Ag}}$ $\left(\mathrm{Mg}_{48} \mathrm{Ag}_{47} \mathrm{Sb}_{48}\right), \mathrm{Ag}_{\mathrm{Mg}}\left(\mathrm{Mg}_{47} \mathrm{Ag}_{49} \mathrm{Sb}_{48}\right), \mathrm{Li}_{\mathrm{Mg}}\left(\mathrm{Mg}_{48} \mathrm{LiAg}_{48} \mathrm{Sb}_{48}\right)$, and $\mathrm{Li}_{\mathrm{Ag}}\left(\mathrm{Mg}_{48} \mathrm{Ag}_{47} \mathrm{LiSb}_{48}\right)$.

$$
\kappa_{\text {min }}=\frac{1}{2}\left[\left(\frac{\pi}{6}\right)^{\frac{1}{3}}\right] k_{B}\left(V^{-\frac{2}{3}}\right)\left(2 \nu_{s}+\nu_{l}\right),
$$

where $V$ is the average volume per atom, and $v_{s}$ and $v_{l}$ are the shear and longitudinal sound velocities, respectively. As a fundamental parameter, the Debye temperature is connected with many physical properties of solids, such as the specific heat, melting point, and elastic constant. At low temperatures, the vibrational excitations arise solely from acoustic vibrations. One of the methods used to calculate the Debye temperature is based on the elastic constant data. The Debye temperature is given by:

$$
\Theta_{D}=\frac{h}{k_{B}}\left[\frac{3 n}{4 \pi}\left(\frac{N_{A} \rho}{M}\right)\right] \nu_{m},
$$

where $k_{B}, h, N_{A}, \rho, M$, and $n$ are the Boltzmann constant, Planck's constant, Avogadro's number, density, molecular weight of the solid, and number of atoms in the molecule, respectively. The average wave velocity $v_{m}$ in polycrystalline materials is approximately given by ${ }^{57}$

$$
\nu_{m}=\left[\frac{1}{3}\left(\frac{2}{\nu_{s}^{3}}+\frac{1}{\nu_{l}^{3}}\right)\right]^{-1 / 3} .
$$

$v_{s}$ and $v_{l}$ can be obtained using the polycrystalline shear modulus $G$ and the bulk modulus $B$ from Navier's equation as follows ${ }^{58}$ :

$$
\nu_{s}=\sqrt{\frac{G}{\rho}} \text { and } \nu_{l}=\sqrt{\frac{B+\frac{4}{3} G}{\rho}},
$$

$B$ and $G$ can estimate using the Voigt-Reuss-Hill approximation from the calculated elastic constant data, which were obtained by the stress-strain method $^{53}$. The calculated elastic constants and the minimum lattice thermal conductivity are listed in Table 1.

For $\alpha$-MgAgSb, $\mathrm{V}_{\mathrm{Ag}}\left(\mathrm{Mg}_{48} \mathrm{Ag}_{47} \mathrm{Sb}_{48}\right), \mathrm{Ag}_{\mathrm{Mg}}\left(\mathrm{Mg}_{47} \mathrm{Ag}_{49} \mathrm{Sb}_{48}\right), \mathrm{Li}_{\mathrm{Mg}}\left(\mathrm{Mg}_{48} \mathrm{LiAg}_{48} \mathrm{Sb}_{48}\right)$, and $\mathrm{Li}_{\mathrm{Ag}}\left(\mathrm{Mg}_{48} \mathrm{Ag}_{47} \mathrm{LiSb}_{48}\right)$, the calculated $\kappa_{\min }$ values are $0.55 \mathrm{~W} / \mathrm{mK}, 0.53 \mathrm{~W} / \mathrm{mK}, 0.54 \mathrm{~W} / \mathrm{mK}, 0.53 \mathrm{~W} / \mathrm{mK}$, and $0.54 \mathrm{~W} / \mathrm{mK}$, respectively. As shown in Eq. 14, the minimum lattice thermal conductivity is strongly affected by the shear sound velocity. Table 1 shows that the $\mathrm{V}_{\mathrm{Ag}}$ and $\mathrm{Li}_{\mathrm{Mg}}$ defects induce an obviously decreasing shear modulus in $\alpha-\mathrm{MgAgSb}$, which indicates that $\mathrm{V}_{\mathrm{Ag}}$ and $\mathrm{Li}_{\mathrm{Mg}}$ defects weaken the resistance against shear deformation of $\alpha-\mathrm{MgAgSb}$. Thus, the shear sound velocity decreases due to $\mathrm{Ag}$ vacancy and $\mathrm{Li}_{\mathrm{Mg}}$ doping. Consequently, the minimum lattice thermal conductivity values are reduced due to $\mathrm{Ag}$ vacancy and $\mathrm{Li}_{\mathrm{Mg}}$ doping. For $\mathrm{Ag} \mathrm{Mg}_{\mathrm{Mg}}$ and $\mathrm{Li}_{\mathrm{Ag}}$ defects, the decrease in the shear modulus is not so large compared with $\mathrm{V}_{\mathrm{Ag}}$ and $\mathrm{Li}_{\mathrm{Mg}}$ defects. Thus, the change in the minimum lattice thermal conductivity due to $\mathrm{Ag}_{\mathrm{Mg}}$ and $\mathrm{Li}_{\mathrm{Ag}}$ defects is smaller than that due to $\mathrm{V}_{\mathrm{Ag}}$ and $\mathrm{Li}_{\mathrm{Mg}}$ defects.

Electrical transport properties. A material with a large ZT needs to have a large $S$ (found in low carrier concentration semiconductors or insulators) and a large $\sigma$ (found in high carrier concentration metals). The carrier concentration dependence of the Seebeck coefficient and the electrical conductivity are shown in Eqs (18) and (19), respectively ${ }^{2}$. In these equations, $T$ is the temperature, and $\mu$ is the charge carrier mobility.

$$
\begin{gathered}
S=\frac{8 \pi k_{B}^{2}}{3 e h^{2}} m_{D O S}^{*} T\left(\frac{\pi}{3 n}\right)^{\frac{2}{3}}, \\
\sigma=n e \mu
\end{gathered}
$$

Equation (18) suggests that the Seebeck coefficient is proportional to the temperature and $m_{D O S}^{*}$, yet is inversely related to the carrier concentration. The electrical conductivity is proportional to the carrier concentration and inversely proportional to the effective mass. We calculated the Seebeck coefficient, $S$, the carrier 


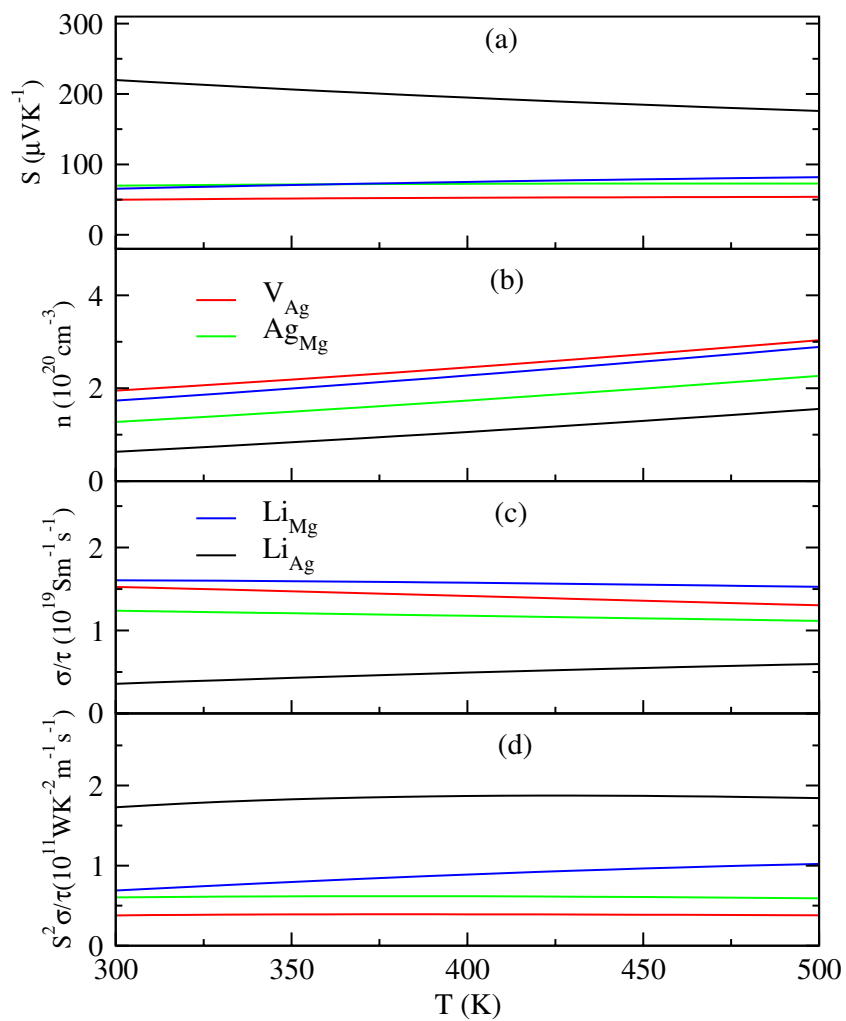

Figure 8. Calculated thermoelectric properties of various defects in $\alpha-\mathrm{MgAgSb}$ as a function of temperature.

concentration, $n$, the electrical conductivity relative to relaxation, $\sigma / \tau$, the thermopower relative to relaxation, and the figure of merit, $S^{2} \sigma / \tau$, as a function of temperature, as shown in Fig. 8.

As can be seen in Fig. 8(a), the Seebeck coefficients of $\mathrm{V}_{\mathrm{Ag}}\left(\mathrm{Mg}_{48} \mathrm{Ag}_{47} \mathrm{Sb}_{48}\right), \mathrm{Ag}_{\mathrm{Mg}}\left(\mathrm{Mg}_{47} \mathrm{Ag}_{49} \mathrm{Sb}_{48}\right), \mathrm{Li}_{\mathrm{Mg}}$ $\left(\mathrm{Mg}_{48} \mathrm{LiAg}_{48} \mathrm{Sb}_{48}\right)$, and $\mathrm{Li}_{\mathrm{Ag}}\left(\mathrm{Mg}_{48} \mathrm{Ag}_{47} \mathrm{LiSb}_{48}\right)$ are all positive over the entire studied temperature range, indicating p-type transport for the four types of defects. The $n$ and $\sigma / \tau$, of $\mathrm{Ag}_{\mathrm{Mg}}$ are lower than those of $\mathrm{V}_{\mathrm{Ag}}$, while $\mathrm{Ag}_{\mathrm{Mg}}$ has the larger $S^{2} \sigma / \tau$ owing to its large $S$. Liu et al. reported that Ag vacancy can be rationally engineered by controlling the hot pressing temperature, and a high peak $Z T$ of $\sim 1.4$ and an average $Z T$ of $\sim 1.1$ can be achieved ${ }^{30}$. $\alpha-\mathrm{MgAgSb}$ containing $\mathrm{Ag}_{\mathrm{Mg}}$ point defects may have higher $Z T$ than with $\mathrm{Ag}$ vacancy because $\alpha-\mathrm{MgAgSb}$ with $\mathrm{Ag}_{\mathrm{Mg}}$ has a larger $S^{2} \sigma / \tau$ than with $\mathrm{Ag}$ vacancy. The $S$ of $\mathrm{Li}_{\mathrm{Ag}}$-doped $\alpha$-MgAgSb is larger than for $\mathrm{Li}_{\mathrm{Mg}}$-doped $\alpha-\mathrm{MgAgSb}$. Although $\mathrm{Li}_{\mathrm{Ag}}$-doped $\alpha$-MgAgSb has the lowest $n$ and $\sigma / \tau$, the $S^{2} \sigma / \tau$ of $\mathrm{Li}_{\mathrm{Ag}}$-doped $\alpha-\mathrm{MgAgSb}$ is larger than that with $\mathrm{Li}_{\mathrm{Mg}}$ defects, due to the large $S$, as shown in Fig. 8(a). Liu et al. found that the average $Z T$ can reach as high as 1.1 from $300 \mathrm{~K}$ to $548 \mathrm{~K}$ when there is Li doping on $\mathrm{Mg}$ sites of $\mathrm{MgAg}_{0.97} \mathrm{Sb}_{0.99}{ }^{39}$. Thus, under more $\mathrm{Mg}$-rich conditions, $\mathrm{Li}_{\mathrm{Ag}}$ doping may lead to a larger $\mathrm{ZT}$ than for $\mathrm{Li}$ substitution on $\mathrm{Mg}$ sites. Thus, good thermoelectric performance as a result of the antisite defect $\mathrm{Ag}_{\mathrm{Mg}}$ and as a result of Li substitution on $\mathrm{Ag}$ sites in $\alpha-\mathrm{MgAgSb}$ can be predicted.

\section{Conclusions}

In this work, we investigated the defect formation energies, the electronic structure, and the thermoelectric performance of the host $\alpha$-MgAgSb and the effects of substitutional Li doping of $\alpha$-MgAgSb, by using density functional theory combined with semiclassical Boltzmann theory. We found that the formation energies strongly depend on the chemical potentials. Ag vacancy and Ag-Mg antisite defects are the dominant defects, acting as the shallow acceptors that determine the $p$-type conduction of experimentally synthesized $\alpha-\mathrm{MgAgSb}$. Moreover, for $\alpha-\mathrm{MgAgSb}$, the $\mathrm{Ag}_{\mathrm{Mg}}$ antisite defect may induce a higher $Z \mathrm{~T}$ than $\mathrm{Ag}$ vacancy, due to the more numerous band valleys near the Fermi level than with $\mathrm{Ag}_{\mathrm{Mg}}$ in $\alpha-\mathrm{MgAgSb} . \alpha-\mathrm{MgAgSb}$ has a secondary valence band with 14 carrier pockets, which indicates that heavily $p$-type doping may lead to a high thermoelectric performance in $\alpha-\mathrm{MgAgSb}$. For Li-doped $\alpha-\mathrm{MgAgSb}$, Li doping on Ag sites has a lower formation energy than on $\mathrm{Mg}$ sites under more $\mathrm{Mg}$-rich conditions, and $\mathrm{Li}_{\mathrm{Ag}}$ may lead to a larger $\mathrm{ZT}$ than for $\mathrm{Li}$ doping on $\mathrm{Mg}$ sites. Thus, engineering atomic scale defects is an effective strategy for enhancing the thermoelectric properties of $\alpha-\mathrm{MgAgSb}$, and the achieved high $Z T$ demonstrates that $\mathrm{Ag}_{\mathrm{Mg}}$ antisite defects and the substitution of $\mathrm{Li}$ on $\mathrm{Ag}$ sites in $\alpha-\mathrm{MgAgSb}$ could lead to materials with good potential for future application in the thermoelectric area.

\section{Computational Details}

The electronic structure of $\alpha-\mathrm{MgAgSb}$ was investigated using the full-potential linearized augmented plane wave $\operatorname{method}^{59}$, as implemented in WIEN2 $\mathrm{k}^{60-62}$. The Tran and Blaha modified semi-local Becke-Johnson exchange correlation potential (TB-mBJ $)^{63}$ was used, which is known to give much more accurate band gaps than the 


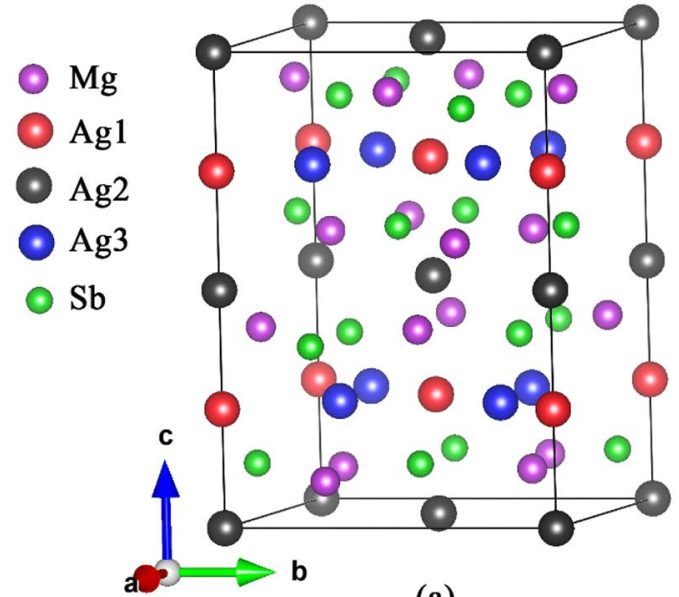

(a)

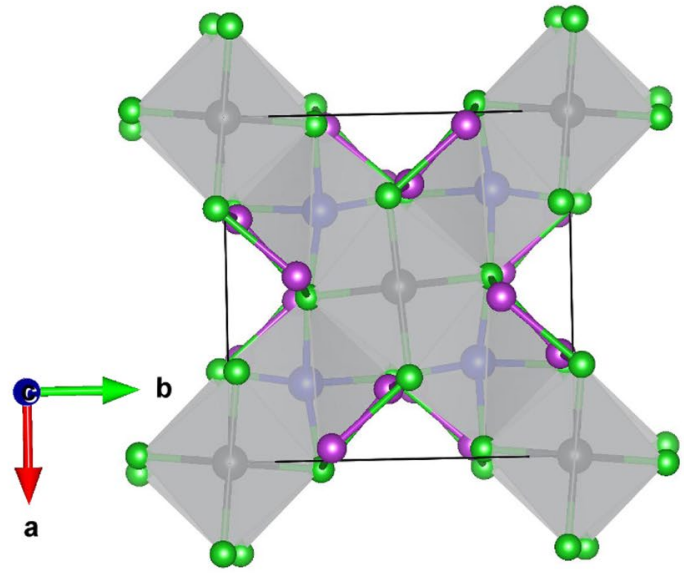

(b)

Figure 9. Crystal structure of $\alpha-\mathrm{MgAgSb}$, looking along the (a) [110] and (b) [001] directions. Mg, Ag1, Ag2, $\mathrm{Ag3}$, and $\mathrm{Sb}$ represent the five crystallographically unique sites of $\alpha-\mathrm{MgAgSb}$.

\begin{tabular}{|l|l|l|l|l|}
\hline Lattice parameter & Atomic type & $\mathbf{x}$ & $\mathbf{y}$ & $\mathbf{z}$ \\
\hline Crystal system: Tetragonal & $\mathrm{Mg}$ & 0.97357 & 0.27610 & 0.11456 \\
\hline Space group: $\mathrm{I} \overline{4} \mathrm{c} 2(\mathrm{NO} .120)$ & $\mathrm{Ag} 1$ & 0.0000 & 0.00000 & 0.25000 \\
\hline$a=9.2816 \AA$ & $\mathrm{Ag} 2$ & 0.0000 & 0.0000 & 0.00000 \\
\hline$c=12.7481 \AA$ & $\mathrm{Ag} 3$ & 0.22158 & 0.22158 & 0.25000 \\
\hline & $\mathrm{Sb}$ & 0.23158 & 0.47522 & 0.11586 \\
\hline
\end{tabular}

Table 2. Lattice constants and atomic coordinates of $\alpha-\mathrm{MgAgSb}$. Ag1, Ag2, and Ag3 represent three crystallographically unique Ag sites.

standard Engel-Vosko generalized-gradient approximation (EV-GGA) ${ }^{64}$. The muffin-tin radii were chosen to be 2.5 a.u. for $\mathrm{Mg}, \mathrm{Ag}$, and $\mathrm{Sb}$. The cut-off parameter $R_{\mathrm{mt}} \times K_{\max }=9$ (where $K_{\max }$ is the magnitude of the largest $k$ vector) was used, and the self-consistent calculations were performed with $2000 \mathrm{k}$-points in the irreducible Brillouin zone; the total energy was made to converge to within $1 \mathrm{mRy}$. The electrical transport properties were then calculated by using semiclassical Boltzmann theory ${ }^{65,66}$ within the constant scattering time approximation, as implemented in the Boltzmann Transport Properties (Boltz-TraP) code ${ }^{67}$. This approximation has been used to calculate the transport coefficients of some known thermoelectric materials and very good agreement with experimental results was achieved ${ }^{68,69}$.

We simulated various defects in $\alpha-\mathrm{MgAgSb}$, along with Li doping, using a supercell that contained 144 atoms. We considered three intrinsic point defects, vacancy, interstitial, and antisite. Because of their large formation energies, cation/anion antisites, such as $\mathrm{Mg}$ or $\mathrm{Ag}$ on the $\mathrm{Sb}$ site and $\mathrm{Sb}$ on the $\mathrm{Mg}$ or $\mathrm{Ag}$ sites, are not discussed in this study. The intrinsic defects considered in this study include $\mathrm{V}_{\mathrm{Ag}}$ (Ag vacancy), $\mathrm{V}_{\mathrm{Mg}}\left(\mathrm{Mg}\right.$ vacancy), $\mathrm{V}_{\mathrm{Sb}}(\mathrm{Sb}$ vacancy), $\mathrm{Mg}_{\mathrm{I}}$ (Mg interstitial), $\mathrm{Ag}_{\mathrm{I}}$ (Ag interstitial), $\mathrm{Sb}_{\mathrm{I}}$ ( $\mathrm{Sb}$ interstitial), $\mathrm{Ag}_{\mathrm{Mg}}$ (Ag on $\mathrm{Mg}$ site), and $\mathrm{Mg}_{\mathrm{Ag}}(\mathrm{Mg}$ on $\mathrm{Ag}$ site). In the case of $\mathrm{Li}$ doping, we simulated interstitial doping $\left(\mathrm{Li}_{\mathrm{I}}\right)$ and substitutional doping, including $\mathrm{Li}$ (Li doping on $\mathrm{Mg}$ site), $\mathrm{Li}_{\mathrm{Ag}}$ ( $\mathrm{Li}$ doping on $\mathrm{Ag}$ site), and $\mathrm{Li}_{\mathrm{Sb}}$ (Li doping on $\mathrm{Sb}$ site).

As shown in Fig. 9, there are 48 atoms in each unit cell of $\alpha-\mathrm{MgAgSb}$, which contains five crystallographically unique atomic sites: one $\mathrm{Mg}$, three $\mathrm{Ag}$, and one $\mathrm{Sb}$. The structural parameters of $\alpha-\mathrm{MgAgSb}$ are shown in Table 2. $\alpha-\mathrm{MgAgSb}$ consists of a distorted Mg-Sb rock-salt lattice, rotated by $45^{\circ}$ about the $c$ axis, with half of the $\mathrm{Mg}-\mathrm{Sb}$ pseudocubes filled with $\mathrm{Ag}$, although the pseudocubes where silver atoms are located are quite different from those in half-Heusler compounds ${ }^{70}$. Such a complex lattice structure may lead to a relatively small thermal conductivity.

We also studied the electronic structure and thermoelectric properties of $\mathrm{V}_{\mathrm{Ag}}, \mathrm{Ag}_{\mathrm{Mg}}, \mathrm{Li}_{\mathrm{Mg}}$, and $\mathrm{Li} \mathrm{ig}_{\mathrm{Ag}}$ using the supercell (144 atoms in MgAgSb supercell), corresponding to a doping level of $2 \%$ for $\alpha-M g A g S b$. We also fixed the lattice constants, only optimizing the internal coordinates. The electronic structures of $\mathrm{Mg}_{48} \mathrm{Ag}_{47} \mathrm{Sb}_{48}$, $\mathrm{Mg}_{47} \mathrm{Ag}_{49} \mathrm{Sb}_{48}, \mathrm{Mg}_{47} \mathrm{LiAg}_{48} \mathrm{Sb}_{48}$, and $\mathrm{Mg}_{48} \mathrm{Ag}_{47} \mathrm{LiSb}_{48}$ were calculated with WIEN2k. Other parameters were in accordance with the calculations for $\alpha-\mathrm{MgAgSb}$.

\section{References}

1. Wood, C. Materials for Thermoelectric Energy Conversion. Rep. Prog. Phys. 51, 459-539, doi:10.1088/0034-4885/51/4/001 (1988).

2. Snyder, G. J. \& Toberer, E. S. Complex Thermoelectric Materials. Nat. Mater. 7, 105-114, doi:10.1038/nmat2090 (2008).

3. Shi, X., Chen, L. \& Uher, C. Recent Advances in High-Performance Bulk Thermoelectric Materials. Int. Mater. Rev. 6, 1-37, doi:10. 1080/09506608.2016.1183075 (2016).

4. Heremans, J. P. et al. Enhancement of Thermoelectric Efficiency in PbTe by Distortion of the Electronic Density of States. Science 321, 554-557, doi:10.1126/science.1159725 (2008). 
5. Zou, T. et al. Enhanced Thermoelectric Performance of $\beta-\mathrm{Zn}_{4} \mathrm{Sb}_{3}$ Based Nanocomposites Through Combined Effects of Density of States Resonance and Carrier Energy Filtering. Sci. Rep 5, 17803, doi:10.1038/srep17803 (2015).

6. Pei, Y. et al. Convergence of Electronic Bands for High Performance Bulk Thermoelectrics. Nature 473, 66-69, doi:10.1038/ nature09996 (2011)

7. Liu, W. et al. Convergence of Conduction Bands as a Means of Enhancing Thermoelectric Performance of $n$-type $\mathrm{Mg}_{2} \mathrm{Si}_{1_{1-x}} \mathrm{Sn}_{\mathrm{x}} \mathrm{Solid}_{\mathrm{d}}$ Solutions. Phys. Rev. Lett. 108, 166601, doi:10.1103/PhysRevLett.108.166601 (2012).

8. Tan, X. J. et al. Multiscale Calculations of Thermoelectric Properties of n-type $\mathrm{Mg}_{2} \mathrm{Si}_{1-\mathrm{x}} \mathrm{Sn}_{\mathrm{x}}$ Solid Solutions. Phys. Rev. B 85, 205212, doi:10.1103/PhysRevB.85.205212 (2012).

9. Fu, C. et al. High Band Degeneracy Contributes to High Thermoelectric Performance in p-Type Half-Heusler Compounds. Adv. Energy Mater. 4, 1400600, doi:10.1002/aenm.201400600 (2014).

10. Wang, H., Gibbs, Z. M., Takagiwa, Y. \& Snyder, G. J. Tuning Bands of PbSe for Better Thermoelectric Efficiency. Energy Environ. Sci. 7, 804-811, doi:10.1039/C3EE43438A (2014).

11. Tang, Y. et al. Convergence of Multi-Valley Bands as the Electronic Origin of High Thermoelectric Performance in $\mathrm{CoSb}_{3}$ Skutterudites. Nat. Mater. 14, 1223-1228, doi:10.1038/nmat4430 (2015).

12. Jian, Z. et al. Significant Band Engineering Effect of YbTe for High Performance Thermoelectric PbTe. J. Mater. Chem. C 3 , 12410-12417, doi:10.1039/C5TC03068D (2015).

13. He, J. et al. Valence Band Engineering and Thermoelectric Performance Optimization in SnTe by Mn-alloying via a Zone-Melting Method. J. Mater. Chem. A 3, 19974-19979, doi:10.1039/C5TA05535K (2015).

14. Fu, C., Zhu, T., Liu, Y., Xie, H. \& Zhao, X. Band engineering of high performance p-type FeNbSb based half-Heusler thermoelectric materials for figure of merit zT >1. Energy Environ. Sci. 8, 216-220, doi:10.1039/C4EE03042G (2015).

15. Zhao, L. D. et al. Ultrahigh Power Factor and Thermoelectric Performance in Hole-Doped Single-Crystal SnSe. Science 351, 141-144, doi:10.1126/science.aad3749 (2016).

16. Yang, D. et al. $\mathrm{Cr}_{2} \mathrm{Ge}_{2} \mathrm{Te}_{6}$ : High Thermoelectric Performance from Layered Structure with High Symmetry. Chem. Mater. 28, 1611-1615, doi:10.1021/acs.chemmater.5b04895 (2016).

17. Heremans, J. P., Thrush, C. M. \& Morelli, D. T. Thermopower Enhancement in Lead Telluride Nanostructures. Phys. Rev. B 70, 115334, doi:10.1103/PhysRevB.70.115334 (2004).

18. Faleev, S. V. \& Léonard, F. Theory of Enhancement of Thermoelectric Properties of Materials with Nanoinclusions. Phys. Rev. B 77, 214304, doi:10.1103/PhysRevB.77.214304 (2008).

19. Walker, C. \& Pohl, R. Phonon Scattering by Point Defects. Phys. Rev 131, 1433-1442, doi:10.1103/PhysRev.131.1433 (1963)

20. Zevalkink, A. et al. Thermoelectric Properties and Electronic Structure of the Zintl Phase $\mathrm{Sr}_{5} \mathrm{In}_{2} \mathrm{Sb}_{6}$ and the $\mathrm{Ca}_{5-\mathrm{x}} \mathrm{Sr}_{\mathrm{x}} \mathrm{In}_{2} \mathrm{Sb}_{6} \mathrm{Solid}$ Solution. J. Phys.: Condens. Matter 27, 015801, doi:10.1088/0953-8984/27/1/015801 (2015).

21. Yang, J., Meisner, G. \& Chen, L. Strain Field Fuctuation Effects on Lattice Thermal Conductivity of ZrNiSn-Based Thermoelectric Compounds. Appl. Phys. Lett. 85, 1140-1142, doi:10.1063/1.1783022 (2004).

22. Snyder, G. J., Christensen, M., Nishibori, E., Caillat, T. \& Iversen, B. B. Disordered Zinc in $\mathrm{Zn}_{4} \mathrm{Sb}_{3}$ with Phonon-Glass and ElectronCrystal Thermoelectric Properties. Nat. Mater. 3, 458-463, doi:10.1038/nmat1154 (2004).

23. Morelli, D. T., Jovovic, V. \& Heremans, J. P. Intrinsically Minimal Thermal Conductivity in Cubic I-V-VI Semiconductors. Phys. Rev. Lett. 101, 035901, doi:10.1103/PhysRevLett.101.035901 (2008).

24. Zhang, Y. et al. First-Principles Description of Anomalously Low Lattice Thermal Conductivity in Thermoelectric Cu-Sb-Se Ternary Semiconductors. Phys. Rev. B 85, 054306, doi:10.1103/PhysRevB.85.054306 (2012).

25. Nielsen, M. D., Ozolins, V. \& Heremans, J. P. Lone Pair Electrons Minimize Lattice Thermal Conductivity. Energy Environ. Sci. 6, 570-578, doi:10.1039/C2EE23391F (2013).

26. Zhang, Y., Ozolinšs, V., Morelli, D. \& Wolverton, C. Prediction of New Stable Compounds and Promising Thermoelectrics in the Cu-Sb-Se System. Chem. Mater. 26, 3427-3435, doi:10.1021/cm5006828 (2014).

27. Zhao, L. D. et al. Ultralow Thermal Conductivity and High Thermoelectric Figure of Merit in SnSe Crystals. Nature 508, 373-377, doi:10.1038/nature13184 (2014)

28. Zhang, Y. First-principles Debye-Callaway approach to lattice thermal conductivity. J. Materiomics 2, 237-247, doi:10.1016/j. jmat.2016.06.004 (2016).

29. Liu, X. et al. Significant Roles of Intrinsic Point Defects in $\mathrm{Mg}_{2} \mathrm{X}(\mathrm{X}=\mathrm{Si}, \mathrm{Ge}, \mathrm{Sn})$ Thermoelectric Materials. Adv. Electron. Mater. 2, 1500284, doi:10.1002/aelm.201500284 (2016).

30. Liu, Z. et al. Understanding and manipulating the intrinsic point defect in $\alpha-\mathrm{MgAgSb}$ for higher thermoelectric performance. J. Mater. Chem. A 4, 16834-16840, doi:10.1039/C6TA06832D (2016).

31. Kirkham, M. J. et al. Ab initio Determination of Crystal Structures of the Thermoelectric Material MgAgSb. Phys. Rev. B 85, 144120, doi:10.1103/PhysRevB.85.144120 (2012).

32. Zhao, H. et al. High Thermoelectric Performance of MgAgSb-based Materials. Nano Energy 7, 97-103, doi:10.1016/j. nanoen.2014.04.012 (2014)

33. Li, D. et al. Atomic Disorders Induced by Silver and Magnesium Ion Migrations Favor High Thermoelectric Performance in

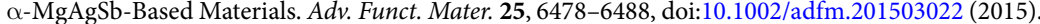

34. Kraemer, D. et al. High Thermoelectric Conversion Efficiency of MgAgSb-Based Material with Hot-Pressed Contacts. Energy Environ. Sci. 8, 1299-1308, doi:10.1039/C4EE02813A (2015).

35. Shuai, J. et al. Study on Thermoelectric Performance by Na Doping in Nanostructured $\mathrm{Mg}_{1-\mathrm{x}} \mathrm{Na}_{\mathrm{x}} \mathrm{Ag}_{0.97} \mathrm{Sb}_{0.99}$. Nano Energy 11, 640-646, doi:10.1016/j.nanoen.2014.11.027 (2015).

36. Sui, J. et al. Effect of Cu Concentration on Thermoelectric Properties of Nanostructured p-type $\mathrm{MgAg}_{0.97-\mathrm{x}} \mathrm{Cu}_{\mathrm{x}} \mathrm{Sb}_{0.99}$. Acta Mater. 87, 266-272, doi:10.1016/j.actamat.2015.01.018 (2015).

37. Miao, N. \& Ghosez, P. Optimization of Thermoelectric Properties of MgAgSb-Based Materials: A First-Principles Investigation. J. Phys. Chem. C 119, 14017-14022, doi:10.1021/acs.jpcc.5b02904 (2015).

38. Ying, P. et al. High Performance $\alpha-M g A g S b$ Thermoelectric Materials for Low Temperature Power Generation. Chem. Mater. 27, 909-913, doi:10.1021/cm5041826 (2015).

39. Liu, Z. et al. Lithium Doping to Enhance Thermoelectric Performance of MgAgSb with Weak Electron-Phonon Coupling. Adv. Energy Mater 6, 1502269, doi:10.1002/aenm.201502269 (2016).

40. Hong, A. et al. Predicting High Thermoelectric Performance of ABX Ternary Compounds $\operatorname{NaMgX}(\mathrm{X}=\mathrm{P}, \mathrm{Sb}, \mathrm{As})$ with Weak Electron-Phonon Coupling and Strong Bonding Anharmonicity. J. Mater. Chem. C 4, 3281-3289, doi:10.1039/C6TC00461J (2016).

41. Liu, Z. et al. Effects of Antimony Content in $\mathrm{MgAg}_{0.97} \mathrm{Sb}_{\mathrm{x}}$ on Output Power and Energy Conversion Efficiency. Acta Mater. 102, 17-23, doi:10.1016/j.actamat.2015.09.033 (2016).

42. Sheng, C. Y. et al. Predicting the Optimized Thermoelectric Performance of MgAgSb. J. Appl. Phys. 119, 195101, doi:10.1063/1.4949479 (2016)

43. Miao, N., Zhou, J., Sa, B., Xu, B. \& Sun, Z. Pressure-induced semimetal-semiconductor transition and enhancement of thermoelectric performance in $\alpha$-MgAgSb. Appl. Phys. Lett. 108, 213902, doi:10.1063/1.4952598 (2016).

44. Liu, Z. et al. The influence of doping sites on achieving higher thermoelectric performance for nanostructured $\alpha-\mathrm{MgAgSb}$. Nano Energy, doi:10.1016/j.nanoen.2016.11.010 (2016).

45. Tang, Y., Hanus, R., Chen, S.-w \& Snyder, G. J. Solubility Design Leading to High Figure of Merit in Low-Cost Ce-CoSb Skutterudites. Nat. Commun. 6, 7584, doi:10.1038/ncomms8584 (2015). 
46. Liu, W.-S. et al. Thermoelectric Property Studies on Cu-Doped n-type $\mathrm{Cu}_{\mathrm{x}} \mathrm{Bi}_{2} \mathrm{Te}_{2.7} \mathrm{Se}_{0.3}$ Nanocomposites. Adv. Energy Mater 1, 577-587, doi:10.1002/aenm.201100149 (2011)

47. Van de Walle, C. G. First-principles calculations for defects and impurities: Applications to III-nitrides. J. Appl. Phys. 95, 3851-3879, doi:10.1063/1.1682673 (2004)

48. Pei, Y., Wang, H. \& Snyder, G. J. Band Engineering of Thermoelectric Materials. Adv. Mater. 24, 6125-6135, doi:10.1002/adma. v24.46 (2012).

49. Goldsmid, H. Thermoelectric Refrigeration. Springer (2013).

50. Ying, P. et al. Hierarchical Chemical Bonds Contributing to the Intrinsically Low Thermal Conductivity in $\alpha-\mathrm{MgAgSb}$ Thermoelectric Materials. Adv. Funct. Mater, doi:10.1002/adfm.201604145 (2016).

51. Wallace, D. C. Thermodynamics of Crystals. Courier Corporation (1998).

52. Wu, Z.-J. et al. Crystal Structures and Elastic Properties of Superhard $\operatorname{IrN}_{2}$ and $\operatorname{IrN}_{3}$ from First Principles. Phys. Rev. B 76, 054115, doi:10.1103/PhysRevB.76.054115 (2007).

53. Hill, R. The Elastic Behaviour of a Crystalline Aggregate. Proc. Phys. Soc. A 65, 349-354, doi:10.1088/0370-1298/65/5/307 (1952).

54. Pugh, S. XCII. Relations Between the Elastic Moduli and the Plastic Properties of Polycrystalline Pure Metals. Lond. Edinb. Dubl. Phil. Mag. 45, 823-843, doi:10.1080/14786440808520496 (1954).

55. Cahill, D. G. \& Pohl, R. O. Lattice Vibrations and Heat Transport in Crystals and Glasses. Annu. Rev. Phys. Chem. 39, 93-121, doi:10.1146/annurev.pc.39.100188.000521 (1988).

56. Cahill, D. G., Watson, S. K. \& Pohl, R. O. Lower Limit to the Thermal Conductivity of Disordered Crystals. Phys. Rev. B 46, 6131-6140, doi:10.1103/PhysRevB.46.6131 (1992).

57. Anderson, O. L. A Simplified Method for Calculating the Debye Temperature from Elastic Constants. J. Phys. Chem. Solids 24, 909-917, doi:10.1016/0022-3697(63)90067-2 (1963).

58. Schreiber, E. \& Orson, L. Elastic Constants and Their Measurement. McGraw-Hill Education (1974).

59. Singh, D. J. \& Nordstrom, L. Planewaves, Pseudopotentials, and the LAPW Method. Springer Science \& Business Media (2006)

60. Hohenberg, P. \& Kohn, W. Inhomogeneous Electron Gas. Phys. Rev 136, B864-B871, doi:10.1103/PhysRev.136.B864 (1964).

61. Koelling, D. D. \& Harmon, B. N. A Technique for Relativistic Spin-Polarised Calculations. J Phys. C: Solid State Phys 10, 3107-3114, doi:10.1088/0022-3719/10/16/019 (1977).

62. Blaha, P., Schwarz, K., Madsen, G., Kvasnicka, D. \& Luitz, J. An Augmented Plane Wave Plus Local Orbital Program for Calculating Crystal Properties. Vienna University of Technology (2001).

63. Tran, F. \& Blaha, P. Accurate Band Gaps of Semiconductors and Insulators with a Semilocal Exchange-Correlation Potential. Phys. Rev. Lett. 102, 226401, doi:10.1103/PhysRevLett.102.226401 (2009).

64. Perdew, J. P., Burke, K. \& Ernzerhof, M. Generalized Gradient Approximation Made Simple. Phys. Rev. Lett. 77, 3865-3868, doi:10.1103/PhysRevLett.77.3865 (1996).

65. Ziman, J. M. Electrons and Phonons: The Theory of Transport Phenomena in Solids. Oxford University Press (1960).

66. Madsen, G. K. H., Schwarz, K., Blaha, P. \& Singh, D. J. Electronic Structure and Transport in Type-I and Type-VIII Clathrates Containing Strontium, Barium, and Europium. Phys. Rev. B 68, 125212, doi:10.1103/PhysRevB.68.125212 (2003).

67. Madsen, G. K. H. \& Singh, D. J. BoltzTraP. A Code for Calculating Band-Structure Dependent Quantities. Comput. Phys. Commun. 175, 67-71, doi:10.1016/j.cpc.2006.03.007 (2006).

68. Shi, Q. F., Yan, Y. L. \& Wang, Y. X. Electronic Structure and Thermoelectric Performance of Zintl Compound $\mathrm{Sr}_{3} \mathrm{GaSb}_{3}$ : A FirstPrinciples Study. Appl. Phys. Lett. 104, 012104, doi:10.1063/1.4860996 (2014).

69. Yan, Y. L. \& Wang, Y. X. Crystal structure, Electronic Structure, and Thermoelectric Properties of $\mathrm{Ca}_{5} \mathrm{Al}_{2} \mathrm{Sb}_{6}$. J. Mater. Chem. 21, 12497-12520, doi:10.1039/cljm11463h (2011).

70. Hirohata, A. et al. Heusler Alloy/Semiconductor Hybrid Structures. Curr. Opin. Solid State Mater. Sci 10, 93-107, doi:10.1016/j. cossms.2006.11.006 (2006).

\section{Acknowledgements}

This research is sponsored by the National Natural Science Foundation of China (nos 11674083, 51571083, 51371076, U1504511, 11547011), the Excellent Youth Foundation of Henan Province (no. 154100510013), the Henan International Science and Technology Cooperation Project (no. 162102410013), the Scheme of Backbone Youth Teachers in the University of Henan Province (2014GGJS-027), and the Key Scientific and Technological Projects in Henan Province (152102210047). All the authors thank Dr. Tania Silver for polishing the English of this paper.

\section{Author Contributions}

Z.F. designed the scheme under the guidance of J.Z., Y.Y., G.Z., C.W. and Y.W. and C.P., F.R. and Z.C. carried out the theoretical analysis. Z.F. carried out the calculations and drafted the manuscript. Y.W. and Z.C. revised the language of the manuscript. All authors reviewed the manuscript.

\section{Additional Information}

Competing Interests: The authors declare that they have no competing interests.

Publisher's note: Springer Nature remains neutral with regard to jurisdictional claims in published maps and institutional affiliations.

Open Access This article is licensed under a Creative Commons Attribution 4.0 International License, which permits use, sharing, adaptation, distribution and reproduction in any medium or format, as long as you give appropriate credit to the original author(s) and the source, provide a link to the Creative Commons license, and indicate if changes were made. The images or other third party material in this article are included in the article's Creative Commons license, unless indicated otherwise in a credit line to the material. If material is not included in the article's Creative Commons license and your intended use is not permitted by statutory regulation or exceeds the permitted use, you will need to obtain permission directly from the copyright holder. To view a copy of this license, visit http://creativecommons.org/licenses/by/4.0/.

(c) The Author(s) 2017 